\title{
Qualidade de vida de transexuais após cirurgia de redesignação sexual
}

\author{
Quality of life of transsexuals after sex reassignment surgery \\ Calidad de vida de los transexuales tras la cirugía de reasignación de sexo
}

Recebido: 05/01/2022 | Revisado: 10/01/2022 | Aceito: 14/01/2022 | Publicado: 16/01/2022

Lucas Siqueira dos Santos ORCID: https://orcid.org/0000-0001-5142-6931

Universidade Tiradentes, Brasil

E-mail: lucascrf648@gmail.com

José Laurindo dos Santos Júnior ORCID: https://orcid.org/0000-0002-8576-4266

Universidade Federal de Sergipe, Brasil

E-mail:juniorsantos.laurindo@gmail.com

Victória Santos Alves

ORCID: https://orcid.org/0000-0001-6122-7952

Universidade Tiradentes, Brasil

E-mail: victorialvesantos@outlook.com

Raquel Santos Alves

ORCID: https://orcid.org/0000-0002-2673-7655

Centro Universitário Maurício de Nassau, Brasil

E-mail: raquel.santos.alves@ outlook.com

Junior de Jesus Guimarães

ORCID: https://orcid.org/0000-0002-4736-6603 Universidade Tiradentes, Brasil

E-mail: juniorguimaraes123@ hotmail.com

Isabella Lorena Souza Silva

ORCID: https://orcid.org/0000-0001-8352-8270

Centro Universitário Maurício de Nassau, Brasil

E-mail: isabella.lorena13@gmail.com

Larissa Ferreira Sales

ORCID: https://orcid.org/0000-0003-4480-9082

Universidade Tiradentes, Brasil

E-mail: larissa.fsales@ souunit.com.br

Carolinne Lisboa Silva

ORCID: https://orcid.org/0000-0003-4747-3123

Centro Universitário Estácio de Sergipe, Brasil

E-mail: carolinnelisboas@gmail.com

Gabriel Vinícius Rabelo dos Santos

ORCID: https://orcid.org/0000-0003-0920-0335

Universidade Tiradentes, Brasil

E-mail: gabriel.vrabelos@gmail.com

Jorge Alfredo Santos

ORCID: https://orcid.org/0000-0002-7626-3873

Universidade Tiradentes, Brasil

E-mail: jorgeafs@ outlook.com

Alice Alexandre Pagan

ORCID: https://orcid.org/0000-0002-9757-4304

Universidade Federal de Sergipe, Brasil

E-mail:apagan22@academico.ufs.br

\section{Resumo}

Este estudo objetivou identificar os benefícios e dificuldades pós-cirúrgicas relacionadas à qualidade de vida de pessoas transexuais após a cirurgia de redesignação sexual. Esta é uma revisão integrativa, realizada entre abril e junho de 2021. Foram levantados artigos nas bases de dados: Biblioteca Virtual da Saúde (BVS), MEDLINE, Scientific Electronic Library Online (SciELO) e PubMed. Essa busca proporcionou a seleção de 12 artigos, publicados entre 2015 e 2020. Após leitura e análise dos objetivos e resultados desses trabalhos, foi possível agrupálos conforme semelhanças, possibilitando na criação de quatro vertentes. São elas: escassez de instrumentos para calcular a qualidade de vida; Complicações pós-cirúrgicas; Importância do acompanhamento multiprofissional pré e pós-cirurgia; Qualidade de vida/satisfação após o procedimento cirúrgico. Em geral, mesmo com algumas complicações pós-operatória, os trabalhos mostraram que o procedimento cirúrgico tem efeitos positivos na vida do paciente, especialmente indicando uma elevada satisfação para questões estéticas e psicossociais após o procedimento. Entretanto, muitos pacientes 
relataram um acompanhamento multiprofissional pós-cirurgia inadequado e escasso. Logo, conclui-se que existem poucos instrumentos para avaliar a qualidade de vida de pessoas transexuais. Além disso, o acompanhamento multiprofissional é de extrema importância no período pré e pós-cirúrgico para evitar possíveis complicações.

Palavras-chave: Qualidade de vida relacionada à saúde; Identidade de gênero; Cirurgia de afirmação de gênero; Transexualidade.

\begin{abstract}
This study aimed to identify the benefits and post-surgical difficulties related to the quality of life of transsexual people after sex reassignment surgery. This is an integrative review, carried out between April and June 2021. Articles were collected in the following databases: Virtual Health Library (VHL), MEDLINE, Scientific Electronic Library Online (SciELO) and PubMed. This search provided the selection of 12 articles, published between 2015 and 2020 . After reading and analyzing the objectives and results of these works, it was possible to group them according to similarities, enabling the creation of four strands. They are: scarcity of instruments to calculate quality of life; Post-surgical complications; Importance of multidisciplinary monitoring before and after surgery; Quality of life/satisfaction after the surgical procedure. In general, even with some postoperative complications, studies have shown that the surgical procedure has positive effects on the patient's life, especially indicating a high satisfaction for aesthetic and psychosocial issues after the procedure. However, many patients reported inadequate and scarce post-surgery multidisciplinary follow-up. Therefore, it is concluded that there are few instruments to assess the quality of life of transsexual people. In addition, multiprofessional follow-up is extremely important in the pre- and post-surgical period to avoid possible complications.
\end{abstract}

Keywords: Health-related quality of life; Gender identity; Gender affirmation surgery; Transsexuality.

\title{
Resumen
}

Este estudio tuvo como objetivo identificar los beneficios y las dificultades posquirúrgicas relacionadas con la calidad de vida de las personas transexuales tras la cirugía de reasignación de sexo. Se trata de una revisión integradora, realizada entre abril y junio de 2021. Los artículos fueron recolectados en las siguientes bases de datos: Biblioteca Virtual en Salud (BVS), MEDLINE, Scientific Electronic Library Online (SciELO) y PubMed. Esta búsqueda arrojó la selección de 12 artículos, publicados entre 2015 y 2020. Después de leer y analizar los objetivos y resultados de estos trabajos, fue posible agruparlos por similitudes, posibilitando la creación de cuatro líneas. Ellos son: escasez de instrumentos para calcular la calidad de vida; Complicaciones posquirúrgicas; Importancia del seguimiento multidisciplinario antes y después de la cirugía; Calidad de vida / satisfacción tras el procedimiento quirúrgico. En general, incluso con algunas complicaciones postoperatorias, los estudios han demostrado que el procedimiento quirúrgico tiene efectos positivos en la vida del paciente, indicando especialmente una alta satisfacción por cuestiones estéticas y psicosociales posteriores al procedimiento. Sin embargo, muchos pacientes refirieron un seguimiento multidisciplinario posquirúrgico inadecuado y escaso. Por tanto, se concluye que existen pocos instrumentos para evaluar la calidad de vida de las personas transexuales. Además, el seguimiento multiprofesional es de suma importancia en el período pre y posquirúrgico para evitar posibles complicaciones.

Palabras clave: Calidad de vida relacionada con la salud; Identidad de género; Cirugía de afirmación de género; Transexualidad.

\section{Introdução}

Transexual é a pessoa que não se identifica com o gênero que lhe foi designado pela sociedade ao nascer, com base em marcadores biológicos. Isso significa dizer que pessoas transexuais passam a se expressar de acordo com o gênero com que se identificam psicologicamente (Barros et al., 2019). O processo transexualizador configura-se como um desafio por refletir diretamente na qualidade de vida das pessoas trans. Elas se deparam frequentemente com barreiras internas, na descoberta da identidade de gênero, ou externas, sob a influência de violências conservadoras tanto da família como da sociedade em geral, impactando negativamente a qualidade de vida (Jorge \& Tavassos, 2018)

O termo qualidade de vida é compreendido por Moreira et al. (2019) como um aspecto amplo, envolvendo o bem-estar físico, espiritual, psicológico e emocional de uma pessoa. Além disso, inclui também elementos sociais, familiares e financeiros (Ferreira et al., 2018).

Nesse sentido, quanto mais positiva a percepção do próprio corpo, maior a satisfação corporal, o que reflete diretamente na qualidade de vida. Isso porque a insatisfação nesse quesito é um potencial motivo para depressão e suicídio (SPIZZIRRI, 2017). 
Mediante esse contexto, frequentemente pessoas transexuais podem desenvolver um mal-estar psíquico, que foi denominado pela medicina como disforia de gênero. Essa condição foi definida pelo $5^{\circ}$ Manual Diagnóstico e Estatístico de Transtornos Mentais [DSM-5] American Psychiatric Association (2014) como um estado de grande desconforto provocado pela inconformidade com o gênero atribuído pela sociedade, associado a quadros de alterações de humor, depressão e ansiedade. Isso implica na qualidade de vida do grupo, atingindo a autoestima e as relações sociais (Morais \& Cortes, 2020).

Embora se concorde parcialmente com essa definição, sobre a ideia de que é comum quadros de sofrimento psicológico associado à pessoa que se identifica como transsexual, é importante enfatizar que os autores e autoras deste trabalho discordam do uso desse termo disforia de gênero, associado à pessoa trans como um tipo de doença, posto que o que gera desconforto psicológico ao grupo são as pressões do mundo dicotômico padronizador cisnormativo que adoeceu na tentativa de fundamentar posições que não reconhecem existência à pessoa trans (Boffi \& Santos, 2020).

Segundo o DMS-5 American Psychiatric Association (2014), cerca de 0,005 a 0,014\% dos homens e 0,002 a 0,003\% das mulheres da população mundial se identificam como transsexuais. Devido a isso, algumas pessoas optam pela realização da cirurgia de redesignação sexual. Esse procedimento reajusta os traços genitais e sexuais do paciente de modo a tornar condizente o corpo com que ele se identifica. Isso é uma alternativa que propõe melhorar a qualidade de vida (Fleury \& Abdo, 2018). Entretanto, é importante ressaltar que nem toda a pessoa transsexual deseja realizar essa intervenção cirúrgica (Fleury \& Abdo, 2018; Boffi \& Santos, 2020).

A redesignação sexual pode aliviar o sofrimento, mas não é o bastante para garantir a qualidade de vida, visto que condutas mais completas devem abordar a saúde em sua complexidade (Morais \& Cortes, 2020). No Brasil, a rede de cuidados ainda é despreparada para oferecer uma assistência efetiva a mulheres trans antes de passarem pela cirurgia de redesignação sexual. Os sistemas de saúde público e privado ainda não são eficientes quanto ao acolhimento e respeito dos direitos das pessoas transexuais (Figueiredo et al., 2017).

Logo, este trabalho se justifica devido à escassez de trabalhos que abordem a qualidade de vida de transexuais após a cirurgia de redesignação, principalmente publicados em forma de revisão integrativa. Bem como considerando a escassez de instrumentos para calcular a qualidade de vida de transexuais, complicações pós-cirúrgicas, importância do acompanhamento multiprofissional pré e pós-cirurgia e a qualidade de vida/satisfação após o procedimento cirúrgico. Além disso, considera-se indispensável a construção de conhecimentos que auxiliem no combate a desinformação e a exclusão da pessoa transexual por profissionais e estudantes da área da saúde.

Dessa forma, buscando uma assistência mais humanizada e respeitando a diversidade, o presente estudo tem por objetivo levantar informações referentes à qualidade de vida de pessoas transexuais após a cirurgia de redesignação sexual, de forma a identificar os benefícios e dificuldades pós-cirúrgicas.

\section{Metodologia}

Este estudo é uma revisão integrativa da literatura que foi realizada entre os meses de abril e junho de 2021. Essa abordagem visa à análise crítica e comparativa das informações levantadas, em busca de semelhanças nos resultados de estudos anteriores para sintetizar e padronizar as informações coletadas à procura de analogias (Santos Junior, 2021; Santos \& Santos, 2021).

Foram utilizadas as bases de dados eletrônicas: Biblioteca Virtual da Saúde (BVS), MEDLINE, Scientific Electronic Library Online (SciELO) e PubMed. Com base nos Descritores em Ciências da Saúde (DECS), foram escolhidas as seguintes palavras-chaves: qualidade de vida, cirurgia de redesignação sexual e transexualidade. Além disso, foi usado o operador logístico 
booleano "AND" entre as seguintes palavras "Qualidade de vida, Cirurgia de Redesignação Sexual, Transexualidade" como estratégia de busca nas bases de dados.

Foram incluídos estudos científicos, publicados entre 2015 e 2021, disponíveis gratuitamente na íntegra em espanhol, inglês ou português, que abordassem a pessoa trans e sua qualidade de vida após cirurgia de redesignação sexual. Foram excluídos artigos cujo título e resumo não correspondessem ao objetivo deste trabalho.

Posteriormente, 56 artigos considerados os mais relevantes pelas bases de dados foram lidos na íntegra para verificar o conteúdo e sua relação com o tema em questão. Após isso, 12 trabalhos foram elegíveis para compor esta análise. Essas publicações foram categorizadas em quatro categorias correspondentes às vertentes de pesquisa conforme semelhanças e analogias em comum encontradas nos resultados dos trabalhos, conforme análise documental e do conteúdo proposto por Bardin (2016). Na Figura 1 está o fluxograma das etapas para seleção do material bibliográfico.

Figura 1. Esquematização da busca metodológica nas bases de dados para elaboração da Tabela 1.

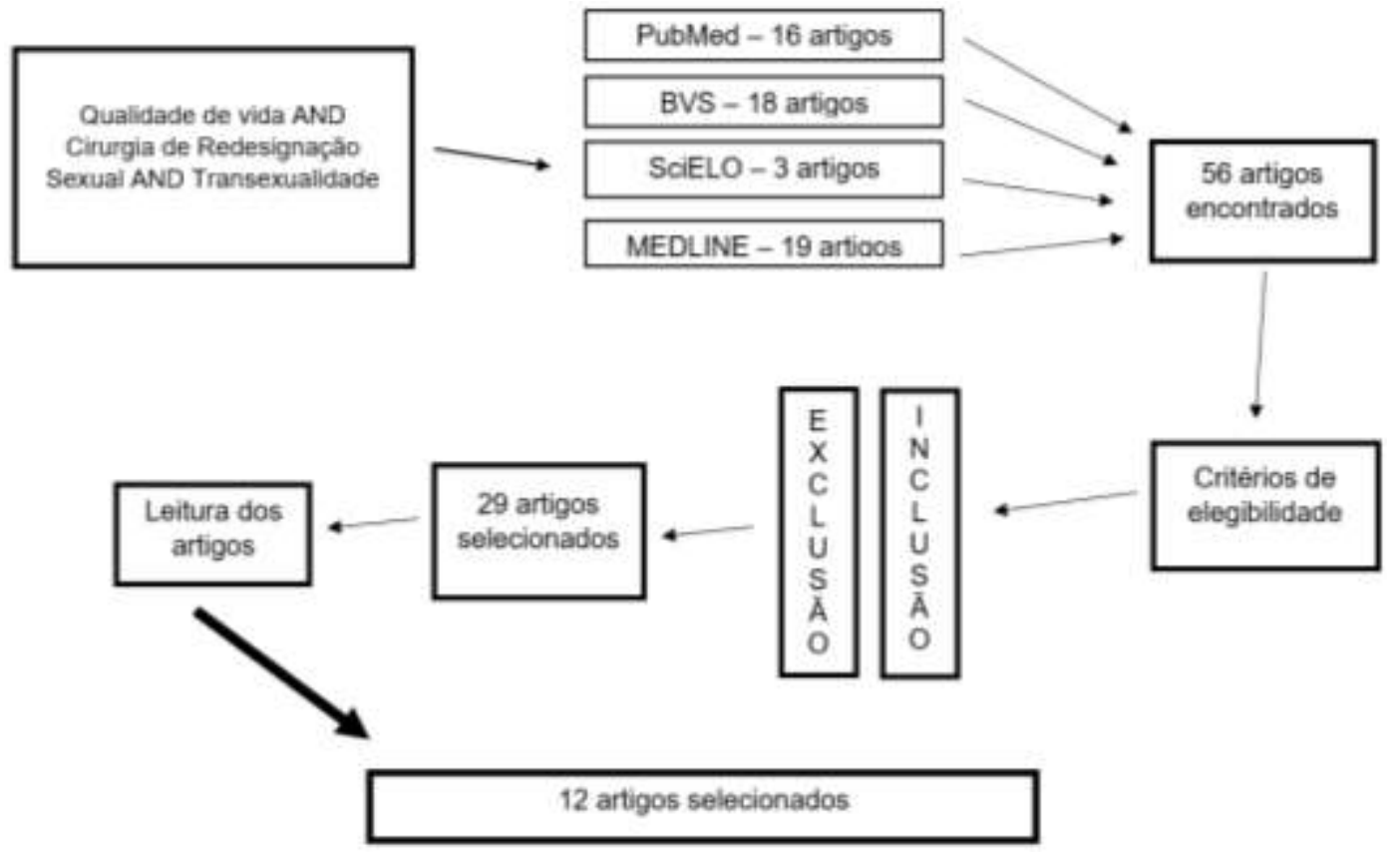

Fonte: Elaborado pelos autores (2021).

Por se tratar de uma pesquisa bibliográfica, os dados obtidos por meio dessa seguiram princípios éticos, segundo a Lei dos Direitos Autorais número 12.853/2013. As informações contidas neste estudo foram citadas de forma fiel, conforme bibliografia selecionada e preconizada pela Associação Brasileira de Normas Técnicas (ABNT) NBR 6023 e NBR 10520.

\section{Resultados}

Ao total, foram utilizadas 12 publicações no estudo, principalmente escritas em inglês (Tabela 1). A maioria dos artigos foram encontrados na base de dados PubMed, seguido pela BVS e SciELO (Tabela 1). Não foi encontrado nenhum artigo relacionado ao tema na base de dados MEDLINE (Tabela 1). 
Tabela 1. Número de publicações (NP) nas bases de dados e idiomas dos artigos científicos relacionados à qualidade de vida de pessoas transexuais após a cirurgia de redesignação sexual.

\begin{tabular}{|c|c|c|c|c|c|c|}
\hline \multirow[b]{2}{*}{ PubMed } & \multicolumn{3}{|c|}{ Base de dados (NP) } & \multicolumn{3}{|c|}{ Idiomas (NP) } \\
\hline & BVS & MEDLINE & SciELO & Português & Espanhol & Inglês \\
\hline 7 & 4 & 0 & 1 & 2 & 1 & 9 \\
\hline
\end{tabular}

Fonte: Dados dos autores e autoras.

A Tabela 2 descreve as informações mais importantes dos estudos selecionados. Após uma leitura e análise associada aos desfechos dos artigos, foi possível visualizar e criar quatro categorias que representam vertentes de pesquisa: 1 - Escassez de instrumentos para calcular a qualidade de vida; 2- Complicações pós-cirúrgicas; 3- Importância do acompanhamento multiprofissional pré e pós-cirurgia; 4- Qualidade de vida/satisfação após o procedimento cirúrgico.

Tabela 2. Principais informações dos artigos científicos levantados relacionados à qualidade de vida de pessoas transexuais após cirurgia de redesignação secundária.

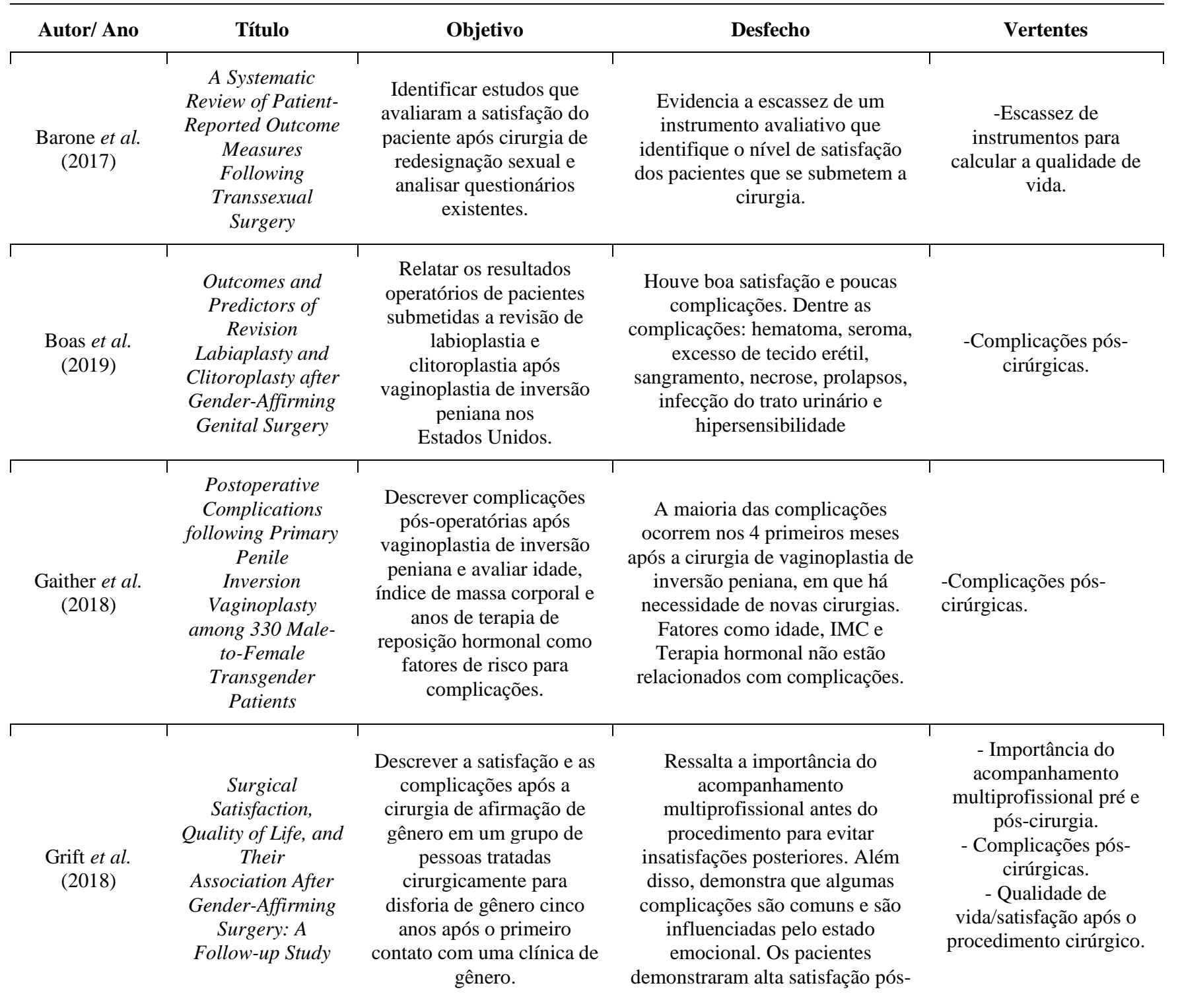




\begin{tabular}{|c|c|c|c|c|}
\hline \multirow[b]{2}{*}{ Herrera et } & \multirow[b]{2}{*}{$\begin{array}{l}\text { Calidad de vida, } \\
\text { función del tracto } \\
\text { urinario y salud } \\
\text { sexual en cirugía } \\
\text { de reasignación de } \\
\text { sexo hombre a } \\
\text { mujer }\end{array}$} & \multirow[b]{2}{*}{$\begin{array}{l}\text { Avaliar a qualidade de } \\
\text { vida, a saúde sexual e a } \\
\text { função do trato urinário } \\
\text { inferior em pacientes com } \\
\text { disforia de gênero } \\
\text { submetidos à cirurgia de } \\
\text { redesignação sexual do } \\
\text { sexo masculino para } \\
\text { feminino. }\end{array}$} & \multicolumn{2}{|l|}{$\begin{array}{l}\text { cirurgia impactando diretamente } \\
\text { na sua qualidade de vida. }\end{array}$} \\
\hline & & & $\begin{array}{l}\text { Demonstra melhoria na qualidade } \\
\text { de vida desses pacientes após a } \\
\text { cirurgia de redesignação sexual, } \\
\text { porém, relata que a satisfação } \\
\text { sexual não é tão bem avaliada, } \\
\text { bem como a aceitação social, logo, } \\
\text { se faz necessário uma avaliação } \\
\text { multiprofissional antes e após o } \\
\text { procedimento. }\end{array}$ & $\begin{array}{l}\text { - Qualidade de } \\
\text { vida/satisfação após o } \\
\text { procedimento cirúrgico. } \\
\text { - Complicações pós- } \\
\text { cirúrgicas. } \\
\text { - Importância do } \\
\text { acompanhamento } \\
\text { multiprofissional pré e } \\
\text { pós-cirurgia. }\end{array}$ \\
\hline $\begin{array}{c}\text { Moraes \& } \\
\text { Cortes }(2020)\end{array}$ & $\begin{array}{l}\text { Cirurgia de } \\
\text { redesignação } \\
\text { sexual: implicações } \\
\text { para o cuidado }\end{array}$ & $\begin{array}{c}\text { Conhecer a produção } \\
\text { científica nacional e } \\
\text { internacional, de } 2007 \text { a } \\
2017 \text {, acerca da cirurgia de } \\
\text { redesignação sexual. }\end{array}$ & $\begin{array}{l}\text { As modificações corporais } \\
\text { cirúrgicas podem influenciar na } \\
\text { melhoria da qualidade de vida e de } \\
\text { fatores psicossociais de pessoas } \\
\text { transexualizadas. }\end{array}$ & $\begin{array}{c}\text { - Complicações pós- } \\
\text { cirúrgicas. } \\
\text { - Qualidade de } \\
\text { vida/satisfação após o } \\
\text { procedimento cirúrgico. }\end{array}$ \\
\hline $\begin{array}{l}\text { Morrison et al. } \\
\quad \text { (2017) }\end{array}$ & $\begin{array}{l}\text { An overview of } \\
\text { female-to-male } \\
\text { gender-confirming } \\
\text { surgery }\end{array}$ & $\begin{array}{c}\text { Fornecer dados atualizados } \\
\text { para orientar profissionais } \\
\text { de saúde na tomada de } \\
\text { decisão baseada em } \\
\text { evidências na cirurgia de } \\
\text { confirmação de gênero. }\end{array}$ & $\begin{array}{l}\text { Afirma a importância da equipe } \\
\text { multidisciplinar no } \\
\text { acompanhamento das } \\
\text { individualidades de cada paciente, } \\
\text { bem como ressalta a insuficiência } \\
\text { de acompanhamentos a longo } \\
\text { prazo dos pacientes que realizam a } \\
\text { cirurgia de redesignação sexual, } \\
\text { para melhor conhecer os níveis de } \\
\text { satisfação desses pacientes. }\end{array}$ & $\begin{array}{c}\text { - Importância do } \\
\text { acompanhamento } \\
\text { multiprofissional pré e } \\
\text { pós-cirurgia. } \\
\text { - Qualidade de } \\
\text { vida/satisfação após o } \\
\text { procedimento cirúrgico. }\end{array}$ \\
\hline $\begin{array}{l}\text { Poudrier et al. } \\
\qquad(2019)\end{array}$ & $\begin{array}{l}\text { Assessing Quality } \\
\text { of Life and Patient- } \\
\text { Reported } \\
\text { Satisfaction with } \\
\text { Masculinizing Top } \\
\text { Surgery: A } \\
\text { Mixed-Methods } \\
\text { Descriptive Survey } \\
\text { Study }\end{array}$ & $\begin{array}{l}\text { Investigar a qualidade de } \\
\text { vida, confiança sexual, } \\
\text { saúde mental, satisfação } \\
\text { com a melhor cirurgia e as } \\
\text { atitudes do paciente em } \\
\text { relação ao papel da melhor } \\
\text { cirurgia na afirmação de } \\
\text { gênero. }\end{array}$ & $\begin{array}{l}\text { Os autores evidenciam a falta de } \\
\text { instrumentos capazes de } \\
\text { acompanhar o nível de satisfação } \\
\text { dos pacientes transgenitalizados a } \\
\text { longo prazo, bem como registrar a } \\
\text { singularidade de cada indivíduo } \\
\text { mediante a experiência. Além } \\
\text { disso, demonstra o êxito na } \\
\text { qualidade de vida, confiança } \\
\text { sexual e estado psicológico desses } \\
\text { pacientes. }\end{array}$ & $\begin{array}{c}\text { - Escassez de } \\
\text { instrumentos para } \\
\text { calcular a qualidade de } \\
\text { vida. } \\
\text { - Qualidade de } \\
\text { vida/satisfação após o } \\
\text { procedimento cirúrgico. }\end{array}$ \\
\hline $\begin{array}{l}\text { Rocon et al. } \\
\quad(2020)\end{array}$ & $\begin{array}{l}\text { Vidas após a } \\
\text { cirurgia de } \\
\text { redesignação } \\
\text { sexual: sentidos } \\
\text { produzidos para } \\
\text { gênero e } \\
\text { Transexualidade }\end{array}$ & $\begin{array}{c}\text { Analisar as compreensões } \\
\text { sobre gênero e } \\
\text { transexualidade bem como } \\
\text { os sentidos para a nova } \\
\text { genitália e para a cirurgia } \\
\text { de redesignação sexual } \\
\text { produzidos por mulheres } \\
\text { que realizaram o referido } \\
\text { procedimento. }\end{array}$ & $\begin{array}{c}\text { Ressalta que a cirurgia de } \\
\text { redesignação sexual por si só não } \\
\text { tem sido suficiente para melhorar a } \\
\text { aceitação de pessoas trans em meio } \\
\text { à sociedade, desse modo, torna-se } \\
\text { imprescindível o acompanhamento } \\
\text { multidisciplinar no processo } \\
\text { transexualizador. }\end{array}$ & $\begin{array}{c}\text { - Importância do } \\
\text { acompanhamento } \\
\text { multiprofissional pré e } \\
\text { pós-cirurgia. } \\
\text { - Qualidade de } \\
\text { vida/satisfação após o } \\
\text { procedimento cirúrgico. } \\
\text { - Complicações pós- } \\
\text { cirúrgicas. }\end{array}$ \\
\hline $\begin{array}{l}\text { Silva et al. } \\
\quad \text { (2016) }\end{array}$ & $\begin{array}{l}\text { WHOQOL-100 } \\
\text { Before and After } \\
\text { Sex Reassignment } \\
\text { Surgery in } \\
\text { Brazilian } \\
\text { Male-to-Female } \\
\text { Transsexual } \\
\text { Individuals }\end{array}$ & $\begin{array}{l}\text { Avaliar o impacto das } \\
\text { intervenções cirúrgicas na } \\
\text { qualidade de vida de } 47 \\
\text { transexuais brasileiros do } \\
\text { sexo masculino para } \\
\text { feminino, utilizando o } \\
\text { WHOQOL-100. }\end{array}$ & $\begin{array}{l}\text { Com o uso do instrumento de } \\
\text { pesquisa WHOQOL-100, o } \\
\text { trabalho mostrou que a qualidade } \\
\text { de vida e as relações sociais de } \\
\text { pessoas que passaram por cirurgia } \\
\text { de redesignação de sexo melhorou } \\
\text { significativamente, porém essas } \\
\text { pessoas continuam a relatar } \\
\text { problemas de saúde física. }\end{array}$ & $\begin{array}{c}\text { - Escassez de } \\
\text { instrumentos para } \\
\text { calcular a qualidade de } \\
\text { vida. } \\
\text { - Complicações pós- } \\
\text { cirúrgicas. }\end{array}$ \\
\hline $\begin{array}{l}\text { Weinfort et al. } \\
\text { (2019) }\end{array}$ & $\begin{array}{l}\text { Quality of Life } \\
\text { Following Male- } \\
\text { To-Female }\end{array}$ & $\begin{array}{l}\text { Fornecer uma visão } \\
\text { detalhada dos dados } \\
\text { atualmente disponíveis } \\
\text { sobre qualidade de vida }\end{array}$ & $\begin{array}{l}\text { Os dados mostram que a cirurgia } \\
\text { de redesignação sexual tem efeitos } \\
\text { positivos em aspectos como: } \\
\text { saúde, bem-estar mental, }\end{array}$ & $\begin{array}{c}\text { - Qualidade de } \\
\text { vida/satisfação após o } \\
\text { procedimento cirúrgico. }\end{array}$ \\
\hline
\end{tabular}




\begin{tabular}{|c|c|c|c|c|}
\hline & $\begin{array}{c}\text { Sex Reassignment } \\
\text { Surgery }\end{array}$ & $\begin{array}{l}\text { após a cirurgia de } \\
\text { redesignação de sexo de } \\
\text { homem para mulher. }\end{array}$ & $\begin{array}{l}\text { sexualidade e satisfação com a } \\
\text { vida em geral. }\end{array}$ & \\
\hline $\begin{array}{l}\text { Papadopulos et } \\
\text { al. (2017) }\end{array}$ & $\begin{array}{l}\text { Male-to-Female } \\
\text { Sex Reassignment } \\
\text { Surgery using the } \\
\text { Combined } \\
\text { Vaginoplasty } \\
\text { Technique: } \\
\text { Satisfaction of } \\
\text { Transgender } \\
\text { Patients } \\
\text { with Aesthetic, } \\
\text { Functional, and } \\
\text { Sexual Outcomes }\end{array}$ & $\begin{array}{l}\text { Avaliar a satisfação do } \\
\text { paciente com os resultados } \\
\text { estéticos, funcionais e } \\
\text { sexuais de MTF SRS } \\
\text { usando nossa técnica de } \\
\text { vaginoplastia combinada. }\end{array}$ & $\begin{array}{l}\text { A satisfação estética e funcional } \\
\text { dos pacientes submetidos à } \\
\text { Cirurgia de Redesignação Sexual } \\
\text { foi bem avaliada mediante uso de } \\
\text { questionários padronizados e } \\
\text { mostrou-se significativa para a } \\
\text { melhoria da qualidade de vida } \\
\text { desses pacientes. }\end{array}$ & $\begin{array}{c}\text { - Escassez de } \\
\text { instrumentos para } \\
\text { calcular a qualidade de } \\
\text { vida. } \\
\text { - Qualidade de } \\
\text { vida/satisfação após o } \\
\text { procedimento cirúrgico }\end{array}$ \\
\hline
\end{tabular}

Fonte: Dados dos autores e autoras.

\section{Discussão}

A primeira cirurgia de redesignação sexual no Brasil foi realizada em 1971, no estado de São Paulo, pelo cirurgião Roberto Farina em uma mulher trans. Naquela época, esse procedimento gerou vários questionamentos preconceituosos que culminaram em um processo por lesão corporal. Em 1974, no IV Congresso de Medicina Legal, essa cirurgia foi conhecida como mutiladora e foi proibida até o ano de 1996, podendo causar processos criminais em médicos que a realizassem (Almendra \& Bertoldi, 2015).

Somente em 1997 o Conselho Federal de Medicina (CFM) elaborou a Resolução no 1.482/97 (Brasil, 1997). Essa autorizava, de forma experimental, a cirurgia de redesignação sexual como neocolpovulvoplastia, neofaloplastia e/ou procedimentos complementares sobre gônadas e caracteres sexuais secundários em pacientes que se enquadrem nos critérios exigidos pela resolução nos hospitais públicos do Brasil. Em 2002, essa resolução foi revogada pela resolução nº 1.652/02 (Brasil, 2010), que autorizou essa intervenção cirúrgica em hospitais públicos e privados, independentemente de ser experimental, visando garantir bem estar às pessoas trans a partir do conceito de saúde adotado pela Organização Mundial de Saúde (OMS). Esse conceito engloba a ausência de patologias, o bem-estar físico, mental e social de uma pessoa (Silva et al., 2019).

Percebe-se que a cirurgia de redesignação influencia positivamente na satisfação pessoal (Silva et al., 2016) promovendo melhoria na qualidade de vida das pessoas transexuais como observado na categoria qualidade de vida/satisfação após o procedimento cirúrgico. (Tabela 2). Contudo, ainda que essa cirurgia impacte significativamente no bem-estar de tais usuários e usuárias, devem ter seus resultados analisados em uma perspectiva global que implique satisfação sexual, social e estética, considerando a complexidade do ser humano (Rocon et al., 2020).

Diante disso, é preciso avaliar as usuárias e usuários que se submetem a esse procedimento cirúrgico, de modo mais amplo. Isso inclui não se limitar à análise dos resultados esperados como o sucesso do procedimento. Envolve também fatores sociais, clínicos e psicológicos, a fim de mensurar o nível de satisfação e, consequentemente, a qualidade de vida dos indivíduos no período pós-cirúrgico (Silva et al., 2019).

Nesse sentido, observa-se que há um aumento significativo na busca pela cirurgia de redesignação sexual. Por exemplo, um estudo norte-americano mostrou que, entre os anos de 2000 e 2011, os índices de procura por intervenções cirúrgicas de redesignação sexual aumentaram 11,9\% [de 72\% para 83,9\%] (Weinforth et al., 2019). Isso se dá, entre outros fatores, devido a busca pelos aspectos essenciais do indivíduo, principalmente o físico, uma vez que esses não se identificam com as características corporais do nascimento (Herrera et al., 2016). 
De mesmo modo, em uma pesquisa realizada na Europa, homens transexuais que não realizaram a cirurgia de mastectomia, relataram qualidade de vida inferior aos que se submeteram à intervenção. Ademais, foi possível identificar uma melhor satisfação quando perceberam o alinhamento do corpo físico com a imagem que traçaram sobre a identificação de gênero. Por outro lado, os poucos resultados sobre a insatisfação daqueles que foram submetidos à redesignação sexual, foram categorizados como morbidade psicológica, desconforto provocado pela falta de bem-estar físico e psicológico que pode impactar diretamente na qualidade de vida, e pouco ou nenhum suporte social (Van De Grift et al., 2018).

A insatisfação em relação a cirurgia envolvendo transexuais se associava a aspectos psicológicos e sociais, sem relação com o desfecho da cirurgia em si. Associado à insatisfação corporal, a possível realização do procedimento refletia, na maioria dos eventos, no receio do rompimento das relações sociais, e do medo de possíveis complicações cirúrgicas (Morais \& Cortes, 2020).

Nessa situação, o contentamento no período pós-cirúrgico dos pacientes que se submetem a cirurgia de redesignação sexual vem acompanhado de melhorias na condição de vida. Como mostrou a vertente complicações pós-cirúrgicas (Tabela 2), isso se perpetua mesmo quando se leva em consideração a ocorrência de complicações ou até mesmo a consistência exaustiva de procedimentos para continuidade da transformação de gênero como, por exemplo, o uso de dilatadores transvaginais ao longo da vida (Silva et al., 2019).

De forma semelhante, de acordo com um estudo realizado no sul do Brasil, mesmo passando pelo risco de complicações operatórias, todas as mulheres da referida pesquisa relataram que fariam tudo de novo pela satisfação de adequarem o corpo à imagem que construíram sobre o gênero ao qual se identificavam. Além disso, 97,35\% delas disseram que poderiam até aconselhar outras mulheres sobre a realização da cirurgia (Morais \& Cortes, 2020).

Em casos de cirurgia de redesignação sexual de pênis para vagina, o procedimento mais comum é o de vaginoplastia de inversão peniana. Esse procedimento visa reproduzir uma vulva com um canal vaginal que permite a penetração, bem como a construção do clítoris e pequenos lábios devidamente funcionais. Apesar do elevado nível de satisfação, na maioria dos casos requer uma reoperação (labioplastia e/ou clitoroplastia) para melhoria estética (Boas et al., 2019). Gaither et al. (2018), observaram que dentre 330 pacientes submetidas à vaginoplastia de inversão peniana, entre os anos de 2011 e 2015, 30 precisaram de uma segunda operação e, entre essas, $90 \%$ eram de labioplastia de revisão.

Boas et al. (2019) com uma amostra de 117 pacientes submetidas a vaginoplastia de inversão peniana, relatam que 54 destas pacientes tiveram algum tipo de complicação pós-cirúrgica. Dentre elas, algumas das principais observadas estão relacionadas a dor prolongada, inchaço e hipersensibilidade local, estenose introital, dispareunia e anorgasmia. Além disso, problemas no trato urinário como: incontinência, fluxo interrompido, infecção e/ou retenção urinária. Ademais, foram relatadas algumas complicações maiores que exigiram internação hospitalar e/ou intervenção cirúrgica, dentre elas: sangramento excessivo, estenose vaginal, fístula retrovaginal, infecções e necrose.

Como compreendido, a cirurgia de redesignação sexual é uma das formas de busca por satisfação com o próprio corpo. Entretanto, em conformidade com o estudo de Schneider et al. (2017), as soluções cirúrgicas são incapazes de resolver traumas psicológicos de imediato, principalmente de eventos preconceituosos consecutivos que a pessoa transexual sofre ao longo da vida. Devido a isso, há necessidade de uma reabilitação mais extensa em todo o acompanhamento, necessário para auxiliar na melhora da qualidade de vida.

Decorrente do processo histórico de lutas por direitos à qualidade de vida, independentemente do gênero ou condição social, em consonância com Popadiuk et al. (2017). Existia até aquele ano de 2017 um esforço do Ministério da Saúde para garantir o direito ao acesso à saúde de qualidade, disponibilizando atendimento multiprofissional a todos os pacientes, inclusive às pessoas transexuais (Popadiuk et al., 2017). 
Entretanto, ainda há dificuldades para promover treinamento aos profissionais para a qualificação da avaliação e monitoramento da saúde desses indivíduos de acordo com suas singularidades, permitindo ofertar uma melhor assistência à saúde, livre de preconceito, respeitando a diversidade sexual do nosso país e garantindo uma assistência de qualidade (Popadiuk et al., 2017).

Diante desse contexto, os estudos evidenciam que o processo transexualizador é complexo e não pode ser analisado apenas partindo de critérios objetivos. Assim, necessita de apoio multiprofissional no pré e pós-cirúrgico para atender, de forma abrangente, às necessidades das usuárias e usuários (Morrrison et al., 2017) como apresentado na vertente importância do acompanhamento multiprofissional pré e pós-cirurgia (Tabela 2).

Nos estudos de Silva et al. (2016) e Papadopulos et al. (2017), questionários foram usados como instrumentos de coleta de dados, constatando que a maioria dos respondentes assinalaram alta satisfação após a cirurgia de redesignação no que se refere a saúde mental e aspectos gerais da vida e da sexualidade. Contudo, alguns pacientes relataram problemas póscirurgia. Esses problemas afetaram parcialmente a qualidade de vida após o procedimento, como mostrou os estudos de Boas et al. (2019) e Van De Grift et al. (2018).

Por fim, alguns pesquisadores criaram instrumentos para mensurar o nível de satisfação, contudo, autores afirmam que há escassez de instrumentos de coleta de dados padronizados por órgãos de saúde que possam avaliar de forma mais fidedigna e a longo prazo o nível de satisfação física, emocional, psicossocial e estética dos pacientes submetidos à cirurgia de redesignação, como observado na vertente escassez de instrumentos para calcular a qualidade de vida como mostra a tabela 2 (Poudrier $\mathrm{et} \mathrm{al.,}$ 2019)

\section{Conclusão}

Percebe-se que há uma escassez significativa na elaboração e aplicação de instrumentos que avaliam a qualidade de vida de pessoas transsexuais relacionados à satisfação sexual, psicossocial e estética. Mesmo com muitas complicações evidenciadas em alguns estudos, essas não são frequentes. Bem como, a satisfação com o procedimento geralmente é elevada. No entanto, autores ressaltam a importância de um acompanhamento psicológico adequado das pessoas interessadas no procedimento, antes mesmo de realizá-lo. Além disso, um acompanhamento multiprofissional rotineiro e duradouro no período pré e pós-cirúrgico, a fim de avaliar com precisão a qualidade de vida de pessoas transexuais submetidas a cirurgia de redesignação.

Sugere-se como trabalhos futuros, através de uma pesquisa de campo, a investigação minuciosa dos impactos positivos e negativos ao longo dos anos da cirurgia de redesignação sexual, além disso, estudar a possibilidade da criação de um instrumento padronizado para calcular a qualidade de vida dessas pessoas.

\section{Referências}

Almendra, R. B. P. G., \& Bertoldi, M. E. (2015). O aparato legal acerca da cirurgia de redesignação sexual e o princípio da dignidade da pessoa humana. JUSFARESC-Revista Jurídica Santa Cruz, 7(7).

American Psychiatric Association. (2014). DSM-5: Manual diagnóstico e estatístico de transtornos mentais. Artmed Editora.

Bardin L. Análise de Conteúdo. Edições 70, 2016.

Barone, M., Cogliandro, A., Di Stefano, N., Tambone, V., \& Persichetti, P. (2017). A systematic review of patient-reported outcome measures following transsexual surgery. Aesthetic plastic surgery, 41(3), 700.

Barros, L. D. O., Lemos, C. R. B., \& Ambiel, R. A. M. (2019). Qualidade de vida e satisfação com a imagem corporal de transexuais. Arquivos Brasileiros de Psicologia, 71(1), 184-195.

Boas, S. R., Ascha, M., Morrison, S. D., Massie, J. P., Nolan, I. T., Shen, J. K., \& Satterwhite, T. (2019). Outcomes and predictors of revision labiaplasty and clitoroplasty after gender-affirming genital surgery. Plastic and reconstructive surgery, 144(6), 1451-1461. 
Boffi, L. C., \& Santos, M. A. Capítulo 1 POSE: Reflexões Acerca Da Construção Do Corpo Travesti. Leituras Sobre A Sexualidade Em Filmes: identidades dissidentes e opressões, 11.

Carmo, F. J. D. E. S. (2020). A intervenção fisioterapêutica na reabilitação pós cirurgia de redesignação de sexo masculino para feminino: relato de caso. Revista Pesquisa em Fisioterapia, 10(2), 288-300.

Conselho Federal de Medicina (CFM). Lei nº. 1.482/97, de 10 de setembro de 1997. Dispõe sobre a cirurgia de redesignação sexual. 10 de set 1997.

Conselho federal de medicina, de 12 agosto de 2010. Dispõe sobre a cirurgia de transgenitalismo e revoga a Resolução CFM nº $1.652 / 02.12$ agosto 2010.

da Silva, D. C., Schwarz, K., Fontanari, A. M. V., Costa, A. B., Massuda, R., Henriques, A. A., \& Lobato, M. I. R. (2016). WHOQOL-100 before and after sex reassignment surgery in Brazilian male-to-female transsexual individuals. The journal of sexual medicine, 13(6), 988-993.

Ferreira, L. K., Meireles, J. F. F., \& Ferreira, M. E. C. (2018). Avaliação do estilo e qualidade de vida em idosos: uma revisão de literatura. Revista Brasileira de Geriatria e Gerontologia, 21, 616-627.

Figueiredo, R., Schwach, K., Wolfe, B. M., McBritton, M., \& Marquezine, I. M. (2017). Mudança de Nome Social de Pessoas Transgêneras: identidade de gênero para além da biologia. Bagoas-Estudos gays: gêneros e sexualidades, 11(17).

Fleury, H. J., \& Abdo, C. H. N. (2018). Atualidades em disforia de gênero, saúde mental e psicoterapia. Diagn Tratamento, 23(4), 147-151.

Gaither, T. W., Awad, M. A., Osterberg, E. C., Murphy, G. P., Romero, A., Bowers, M. L., \& Breyer, B. N. (2018). Postoperative complications following primary penile inversion vaginoplasty among 330 male-to-female transgender patients. The Journal of urology, 199(3), 760-765.

Herrera, J. M. S., Soto, J. C., Monroy, S. F., Niño, J. P., Ramirez, G. A. B., Rincon, C. F., \& Sandoval, G. P. (2016). Calidad de vida, función del tracto urinario y salud sexual en cirugía de reasignación de sexo hombre a mujer. Urología colombiana, 25(2), 81-87.

Jorge, M. A. C., \& Travassos, N. P. (2018). Transexualidade: o corpo entre o sujeito e a ciência (Vol. 1). Editora Schwarcz-Companhia das Letras.

Morais, A. V. C., \& Cortes, H. M. (2020). Cirurgia de redesignação sexual: implicações para o cuidado. J. nurs. health, 20103002-20103002.

Moreira, N. B., Mazzardo, O., Vagetti, G. C., de Oliveira, V., \& de Campos, W. (2019). Qualidade de vida: comparação entre sexos e índice de massa corporal em atletas do basquetebol master brasileiro. Revista Brasileira De Educação Física E Esporte, 33(1), 107-114.

Morrison, S. D., Chen, M. L., \& Crane, C. N. (2017). An overview of female-to-male gender-confirming surgery. Nature Reviews Urology, 14(8), 486-500.

Papadopulos, N. A., Zavlin, D., Lellé, J. D., Herschbach, P., Henrich, G., Kovacs, L., ... \& Schaff, J. (2017). Male-to-female sex reassignment surgery using the combined technique leads to increased quality of life in a prospective study. Plastic and reconstructive surgery, 140(2), 286-294.

Peixoto, M. M., \& Nobre, P. (2016). Automatic thoughts during sexual activity, distressing sexual symptoms, and sexual orientation: Findings from a web survey. Journal of sex \& marital therapy, 42(7), 616-634.

Popadiuk, G. S., Oliveira, D. C., \& Signorelli, M. C. (2017). A Política Nacional de Saúde Integral de Lésbicas, Gays, Bissexuais e Transgêneros (LGBT) e o acesso ao processo transexualizador no Sistema Único de Saúde (SUS): avanços e desafios. Ciência \& Saúde Coletiva, 22, 1509-1520.

Poudrier, G., Nolan, I. T., Cook, T. E., Saia, W., Motosko, C. C., Stranix, J. T., \& Hazen, A. (2019). Assessing quality of life and patient-reported satisfaction with masculinizing top surgery: a mixed-methods descriptive survey study. Plastic and reconstructive surgery, 143(1), 272-279.

Rocon, P. C., Sodré, F., Rodrigues, A., Barros, M. E. B. D., Pinto, G. S. S., \& Roseiro, M. C. F. B. (2020). Vidas após a cirurgia de redesignação sexual: sentidos produzidos para gênero e transexualidade. Ciência \& Saúde Coletiva, 25, 2347-2356.

Santos Júnior, J. L., dos Santos, L. S., Meiado, M. V., \& da Silva, E. C. (2021). Recursos didáticos para o processo de ensino-aprendizagem de conteúdos botânicos para educação básica no Brasil. Research, Society and Development, 10(13), e448101321500-e448101321500.

Santos, L. E. S., \& dos Santos, L. S. (2021). O impacto do coronavírus em pacientes cardiopatas. Research, Society and Development, 10(5), e0110514539e0110514539.

Schneider, M. A., Andreazza, T., Fontanari, A. M. V., Costa, A. B., Silva, D. C. D., Aguiar, B. W. D., \& Lobato, M. I. R. (2017). Serum concentrations of brainderived neurotrophic factor in patients diagnosed with gender dysphoria undergoing sex reassignment surgery. Trends in psychiatry and psychotherapy, 39, 4347.

Silva, M. J. D. S., Schraiber, L. B., \& Mota, A. (2019). O conceito de saúde na Saúde Coletiva: contribuições a partir da crítica social e histórica da produção científica. Physis: Revista de Saúde Coletiva, 29.

SpizzirriI, G. (2017). Disforia de gênero em indivíduos transexuais adultos: aspectos clínicos e epidemiológicos. CEP, 1060, 970.

Weinforth, G., Fakin, R., Giovanoli, P., \& Nuñez, D. G. (2019). Quality of life following male-to-female sex reassignment surgery. Deutsches Ärzteblatt International, 116(15), 253 